\title{
A generalized tagging method
}

\author{
V. Grewe \\ DLR-Institut für Physik der Atmosphäre, Oberpfaffenhofen, 82234 Wessling, Germany \\ Correspondence to: V. Grewe (volker.grewe@dlr.de)
}

Received: 12 September 2012 - Published in Geosci. Model Dev. Discuss.: 18 October 2012

Revised: 7 January 2013 - Accepted: 15 January 2013 - Published: 19 February 2013

\begin{abstract}
The understanding of causes of changes in climate-chemistry simulations is an important, but often challenging task. In atmospheric chemistry, one approach is to tag species according to their origin (e.g. emission categories) and to inherit these tags to other species during subsequent reactions. This concept was recently employed to calculate the contribution of atmospheric processes to temperature. Here a new concept for tagging any state variable is presented. This generalized tagging method results from a sensitivity analysis of the individual forcing terms of the right hand side of the governing differential equations. In a couple of examples, the consistency with previous approaches and the synergy by using different analysis techniques are shown. Since the method is based on a ratio describing relative sensitivities, singularities occur where the method is not applicable. For some applications, such as in atmospheric chemistry, these singularities can easily be removed. However, one theoretical example is given, where this method is not applicable at all.
\end{abstract}

\section{Introduction}

In order to answer questions, such as: "what is the contribution of road traffic emissions to climate change?" and "what is the contribution of anthropogenic $\mathrm{CO}_{2}$ emissions to climate change?", climate (-chemistry) models were applied to provide an answer (Uherek et al., 2010; IPCC, 2007). Most applications rely on the assessment of changes, i.e. the sensitivity of the atmosphere to a change in a regarded quantity, such as road traffic emissions. Such a comparison of two experiments, one including all emissions, processes, etc. and one where the regarded process is altered (e.g. road traffic emissions suppressed), provides valuable information on the sensitivity of, e.g. the atmosphere to road traffic emissions.
However, it was shown that this perturbation approach does not provide a reliable estimate of the contribution of these emissions to ozone and climate change (Wang et al., 2009; Grewe et al., 2010, 2012; Emmons et al., 2012). For example, when ozone production is saturated, i.e. additional $\mathrm{NO}_{\mathrm{x}}$ molecules only lead to a very low additional ozone production, this very low ozone production is applied in the perturbation method to estimate the total ozone produced.

On the contrary, the tagging methodology provides a useful framework to obtain information on the contribution of individual processes to specific quantities. It is widely used in atmospheric chemistry, but only recently well documented (Grewe, 2004, 2012; Wang et al., 2009; Gromov et al., 2010; Grewe et al., 2010; Butler et al., 2011; Emmons et al., 2012). In most applications, a subset of species is regarded and tagged without non-linear interaction between the individual species. For example road traffic emissions and the contributions to nitrogen oxide and ozone concentrations are tagged, but the interaction of, e.g. road traffic nitrogen oxides emissions and ship traffic non-methane hydrocarbon emissions is not regarded. Grewe et al. (2010) provided a methodology, which allows a complete tagging of a chemical scheme and takes these non-linear interdependencies into account.

In the past, many diagnostics were developed and applied to understand the non-linear chemistry-atmosphere system and its response to a perturbation. The analysis of individual reactions and reaction chains and the analysis of local ozone production and loss changes were frequently used. Other studies have analysed the linearization of a perturbation of a (steady-state) solution. For example, Prather (1998) investigated the chemical modes (decay times of a perturbation) of a reduced system, which includes the three species ozone, methane and nitrous oxide. Maas and Pope (1992) identified in a more theoretical framework, manifolds for the linearized system, which contain chemistry with slow response times, 
and can therefore be used to simplify chemical kinetics. Both studies are targeting at the characterization of response times or chemical modes of a perturbation. Tagging on the other hand is not primarily focusing on a perturbation nor does it include a linearization of the system. Instead, it is merely a complex budget analysis and a source-receptor relationship, where the source can be defined very arbitrarily, e.g. emissions at different regions, emissions from different categories (road traffic, ships, biomass burning, etc.) or from various processes. The combination of all these analysis techniques leads to a better insight in interactions within non-linear systems.

Recently, this methodology was applied to a simple climate-box model to investigate the impact of atmospheric absorption on surface temperature, i.e. the greenhouse effect (Grewe, 2012). Hence it shows that in principle the tagging methodology is also applicable to quantities other than only chemical species.

Here, these approaches are generalized to provide a framework with which any quantity can be tagged. This generalized tagging approach is introduced in Sect. 2. Section 3 provides four examples, which show how this formalism can be applied. These examples prove the consistency with previous approaches in Grewe et al. (2010) and Grewe (2012), which were derived with a different, combinatorial, ansatz. The applicability of this methodology is limited by the existence of singularities, which are explored in more detail in Sect. 4. Section 5 provides an example of a simple non-linear system and how different analysis techniques can be combined.

\section{A generalized tagging method}

In a very generalized form, climate-chemistry models describe the temporal development of $n$ state variables $x_{i}$, $i=1, . ., n$, which can be written in vector form: $x^{\mathrm{T}}=$ $\left(x_{1}, \ldots, x_{n}\right) \in \mathbb{R}^{n}$. (An overview and summary of the definition of the main variables is given in Table 1). This temporal evolution is given by differential equations, describing the dependence on external forcings $\boldsymbol{P}(t)^{\mathrm{T}}=$ $\left(P_{1}(t), \ldots, P_{n}(t)\right) \in \mathbb{R}^{n}$ and on the state variables themselves $\boldsymbol{F}(\boldsymbol{x})^{\mathrm{T}}=\left(F_{1}(\boldsymbol{x}), \ldots, F_{n}(\boldsymbol{x})\right) \in \mathbb{R}^{n}:$

$\frac{\partial}{\partial t} \boldsymbol{x}=\boldsymbol{P}(t)+\boldsymbol{F}(\boldsymbol{x})$.

In general, $\boldsymbol{F}(\boldsymbol{x})$ describes a sum of individual processes, such as various reactions. Without loss of generality, this is restricted to one process only. The method described below can then be applied to each summand individually.

Now, we are interested in following the contributions of individual processes or quantities to the state variables. For example in atmospheric chemistry applications the contribution of emissions (here $\boldsymbol{P}(t)$ ) to the atmospheric composition (here $\boldsymbol{x}$ ) is of interest. Another example is climate modelling: the contribution of greenhouse gases to temperature. Hence we define $m$ categories, which totally partition the right hand side and, as a consequence also the left hand side of Eq. (1):

$$
\begin{aligned}
P_{i}(t) & =\sum_{j=1}^{m} P_{i}^{j}(t) \\
F_{i}(\boldsymbol{x}) & =\sum_{j=1}^{m} F_{i}^{j}(\boldsymbol{x}) \\
\Rightarrow x_{i} & =\sum_{j=1}^{m} x_{i}^{j} .
\end{aligned}
$$

Obviously, the challenging part of the decomposition is to derive the terms $F_{i}^{j}(\boldsymbol{x})$. In atmospheric chemistry a combinatorial approach was chosen to derive these terms (Grewe et al., 2010). That means that for a reaction of, e.g. species $x_{1}$ with species $x_{2}$, every possible combination of the categories $j$ and $k$ of either species was calculated. Hence all possible combinations of the contributions $x_{1}^{j}$ and $x_{2}^{k}$ of species $x_{1}$ and $x_{2}$ were calculated. Here, a different approach is chosen. In Sect. 3 it is shown that both approaches are equivalent and lead to identical results.

The basic question is "what is the impact of category $j$ on the term $F_{i}(\boldsymbol{x})$ ?" The impact is defined as the sensitivity of the category $j$ on the right hand side multiplied by state variable of category $j$. Or in other words, the impact $Q_{i}^{j}$ of category $j$ for the term $F_{i}$ is

$Q_{i}^{j}=\boldsymbol{x}^{j^{\mathrm{T}}} \frac{\partial F_{i}(\boldsymbol{x})}{\partial \boldsymbol{x}^{j}}$,

where $\boldsymbol{x}^{j}=\left(x_{1}^{j}, \ldots, x_{n}^{j}\right)^{\mathrm{T}}$, the contribution of category $j$ to $x$. The total impact $T_{i}$ of all categories is then

$T_{i}=\sum_{j=1}^{m} Q_{i}^{j}$.

And the contribution $F_{i}^{j}(\boldsymbol{x})$ of the category $j$ to $F_{i}(\boldsymbol{x})$ is the relative contribution of category $j$ :

$F_{i}^{j}(\boldsymbol{x})=\frac{Q_{i}^{j}}{T_{i}} F_{i}(\boldsymbol{x})$

The differential equations for the tagged quantities $x_{i}^{j}$ are then:

$$
\begin{aligned}
\frac{\partial}{\partial t} x_{i}^{j} & =P_{i}^{j}(t)+F_{i}^{j}(\boldsymbol{x}) \\
& =P_{i}^{j}(t)+\frac{Q_{i}^{j}}{T_{i}} F_{i}(\boldsymbol{x}) .
\end{aligned}
$$

In vector notation and with $\frac{\partial x}{\partial x_{i}}=\frac{\partial \sum_{j=1}^{m} x_{j}}{\partial x_{i}}=\frac{\partial x_{i}}{\partial x_{i}}=1$ follows:

$$
\frac{\partial}{\partial t} x_{i}^{j}=P_{i}^{j}(t)+F_{i}(\boldsymbol{x}) \frac{\boldsymbol{x}^{j^{\mathrm{T}}} \nabla F_{i}(\boldsymbol{x})}{\boldsymbol{x}^{\mathrm{T}} \nabla F_{i}(\boldsymbol{x})} .
$$


Table 1. Overview on used variables. The time dependency " $(t)$ " is occasionally omitted for simplicity reasons, e.g. $\boldsymbol{x}(t)$ becomes $\boldsymbol{x}$.

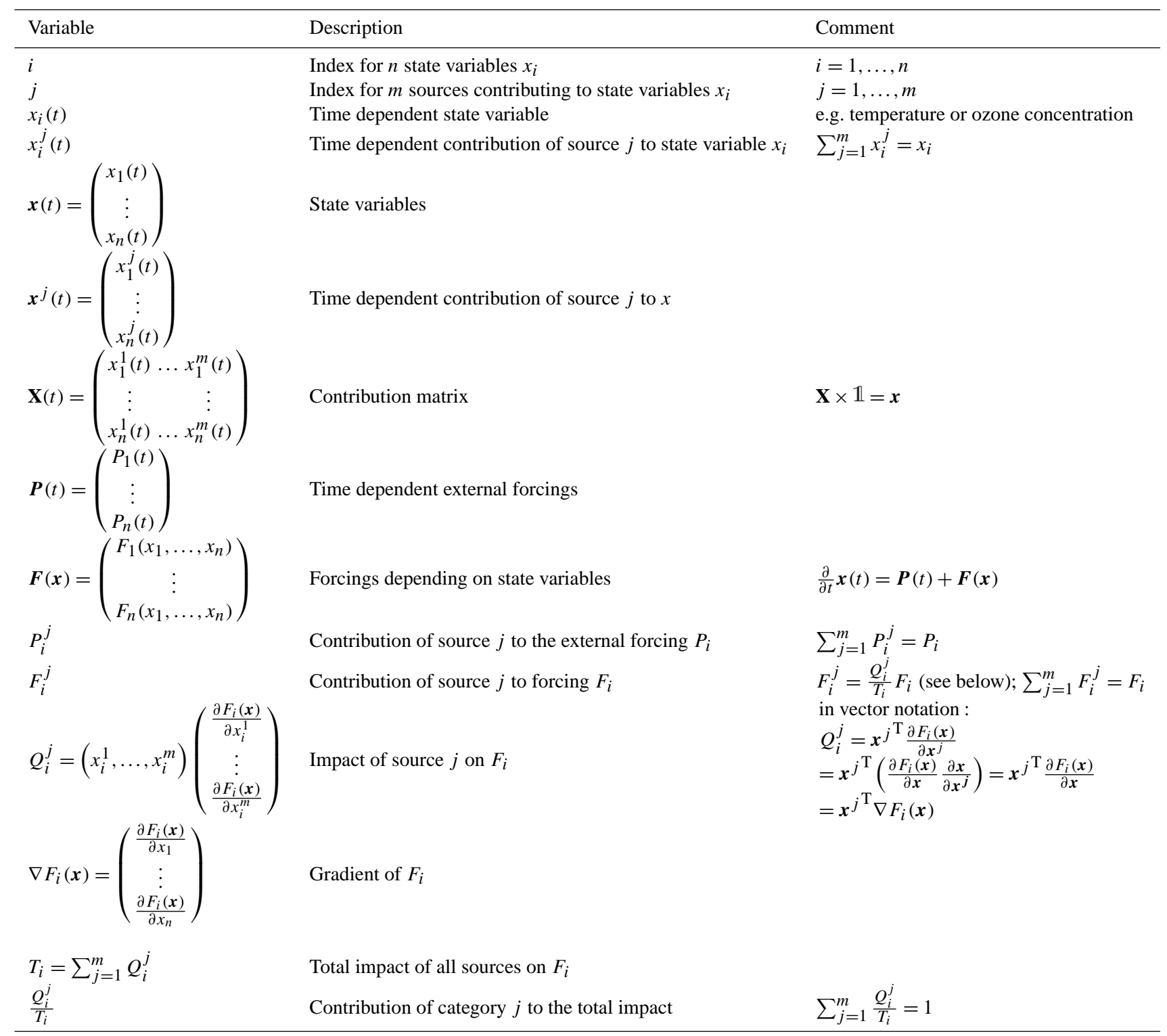

Obviously, the method has a singularity and hence needs special treatments for situations where $\boldsymbol{x}^{\mathrm{T}} \nabla F_{i}(\boldsymbol{x})=0$. Before this singularity will be discussed in more detail, examples will be given to obtain a better understanding of the practical consequences of Eq. (10) and the consistency with previous tagging approaches in Grewe et al. (2010) and Grewe (2012) will be shown.

\section{Examples}

\subsection{Self-dependency}

In many cases the regarded quantity $x_{i}$ depends only on a temporal forcing and on itself, i.e. $\boldsymbol{F}(\boldsymbol{x})=$

$$
\begin{gathered}
\left(0, \ldots, 0, f\left(x_{i}\right), 0, \ldots, 0\right)^{\mathrm{T}}: \\
\frac{\partial}{\partial t} x_{i}^{j}=P_{i}^{j}(t)+f\left(x_{i}\right) \frac{x_{i}^{j} f^{\prime}\left(x_{i}\right)}{x_{i} f^{\prime}\left(x_{i}\right)} \\
=P_{i}^{j}(t)+f\left(x_{i}\right) \frac{x_{i}^{j}}{x_{i}}
\end{gathered}
$$

This means that the right hand forcing term is linearly decomposed into the contributions according to the contributions of the state variable $x_{i}^{j}$.

Examples are tracers, such as Radon, which are emitted at the Earth's surface $\left(P_{i}^{j}\right)$ and decay radioactively, i.e. $f\left(x_{i}\right)=$ $-\frac{x_{i}}{\tau}$, where $\tau$ is the lifetime of the regarded species. 
Table 2. Overview on the characteristics of the non-linear system $\dot{x}=P-x^{\alpha}$. See Sect. 5 for details.

\begin{tabular}{lll}
\hline Description & Variable & Result \\
\hline Steady-state solution & $x^{\mathrm{s}}$ & $=P^{\frac{1}{\alpha}}=P \tau_{x}$ \\
Lifetime of $x$ & $\tau_{x}$ & $=P^{\frac{1-\alpha}{\alpha}}$ \\
Lifetime of a perturbation $y$ to $x \mathrm{~s}$ & $\tau_{y}$ & $=\alpha P^{\frac{1-\alpha}{\alpha}}=\alpha \tau_{x}$ \\
Steady-state solution of tagged quantity & ${ }^{t} x_{i}^{\mathrm{s}}$ & $=P_{i} P^{\frac{1-\alpha}{\alpha}}=P_{i} \tau_{x}$ \\
Contribution calculated with perturbation approach & $\mathrm{p}_{x_{i}^{\mathrm{s}}}^{\mathrm{s}}$ & $=\frac{1}{\alpha} P_{i} P^{\frac{1-\alpha}{\alpha}}=P_{i} \tau_{y}$ \\
\hline
\end{tabular}

\subsection{Bimolecular reactions and similar processes}

Here a reaction of two species is regarded:

$x_{1}+x_{2} \longrightarrow x_{3}$,

e.g. the reaction of $\mathrm{NO}$ and $\mathrm{HO}_{2}$, which forms $\mathrm{OH}$ and $\mathrm{NO}_{2}$ and latter photolyses and recombines to $\mathrm{O}_{3}$. Therefore, we consider three species only, i.e. $n=3$. The differential equation for the production of $x_{3}$ by this equation is

$\frac{\partial}{\partial t} x_{3}=F_{3}(\boldsymbol{x})=k x_{1} x_{2}$

with $k$ the reaction rate coefficient.

In this case $F_{3}(\boldsymbol{x})=k x_{1} x_{2}$ with the notation of Eq. (10). With $m$ arbitrary categories, the differential equation for the tagged species $x_{3}^{j}(j=1, \ldots, m)$ becomes

$$
\begin{aligned}
\frac{\partial}{\partial t} x_{3}^{j} & =F_{3}(\boldsymbol{x}) \frac{\left(x_{1}^{j}, x_{2}^{j}, x_{3}^{j}\right)\left(k x_{2}, k x_{1}, 0\right)^{\mathrm{T}}}{\left(x_{1}, x_{2}, x_{3}\right)\left(k x_{2}, k x_{1}, 0\right)^{\mathrm{T}}} \\
& =F_{3}(\boldsymbol{x}) \frac{k x_{1}^{j} x_{2}+k x_{2}^{j} x_{1}}{2 k x_{1} x_{2}} \\
& =F_{3}(\boldsymbol{x}) \frac{1}{2}\left(\frac{x_{1}^{j}}{x_{1}}+\frac{x_{2}^{j}}{x_{2}}\right) .
\end{aligned}
$$

Therefore the contribution of the category $j$ to the production of $x_{3}^{j}$ is determined by the mean contribution of the educts, i.e. $1 / 2\left(x_{1}^{j} / x_{1}+x_{2}^{j} / x_{2}\right)$. Hence it is identical to results in Grewe et al. (2010).

\subsection{Ternary and multi-body reactions}

Let us now consider a chemical reaction, where $(m-1)$ educts lead to the species $x_{m}$ :

$x_{1}+\ldots+x_{m-1} \longrightarrow x_{m}$.

The differential equation for the production is then

$\frac{\partial}{\partial t} x_{m}=F_{m}(\boldsymbol{x})=k_{m} \prod_{i=1}^{m-1} x_{i}$

where $k_{m}$ is the reaction rate coefficients.
The derivative of the right hand side gives

$$
\frac{\partial}{\partial x_{i}} F_{m}(\boldsymbol{x})=k_{m} \prod_{k=1, k \neq i}^{m-1} x_{k}=\frac{F_{m}(\boldsymbol{x})}{x_{i}}
$$

And therefore, according to Eq. (10), the differential equation for the contribution results in

$$
\begin{aligned}
\frac{\partial}{\partial t} x_{m}^{j} & =F_{m}(\boldsymbol{x}) \frac{\sum_{i=1}^{m-1} x_{i}^{j} \frac{\partial}{\partial x_{i}} F_{m}(\boldsymbol{x})}{\sum_{i=1}^{m-1} x_{i} \frac{\partial}{\partial x_{i}} F_{m}(\boldsymbol{x})} \\
& =F_{m}(\boldsymbol{x}) \frac{\sum_{i=1}^{m-1} \frac{x_{i}^{j}}{x_{i}} F_{m}(\boldsymbol{x})}{\sum_{i=1}^{m-1} F_{m}(\boldsymbol{x})} \\
& =F_{m}(\boldsymbol{x}) \frac{1}{m-1} \sum_{i=1}^{m-1} \frac{x_{i}^{j}}{x_{i}} .
\end{aligned}
$$

This means that the contribution of category $j$ to the production of a species $x_{m}$ by a $(m-1)$-body chemical reaction is the mean of the contributions of the individual species (educts) $x_{i}$ to category $j$. This is again consistent with the previously derived equations for, e.g. a ternary reaction (Grewe et al., 2010).

\subsection{Heating rates}

Here an example is given for an alternative numerical approach for calculating the right hand side of the tagging equation Eq. (10). In the case of a tagging of temperatures (Grewe, 2012), the temperature equation includes diabatic terms and one of them is the diabatic heating rates from radiative processes. Instead of going through the math in the radiation calculation to derive the right hand side of the tagging equations, it is also possible to additionally calculate the heating rates with perturbations for each of the $m$ categories.

Assume that the radiative transfer code provides vertical profiles in heating rates $H(z)$ (in $\mathrm{Ks}^{-1}$ ) for an altitude coordinate $z$ at any given location. For simplicity reasons, we assume only one state variable temperature $T$ and two categories, which influence temperature, greenhouses gases 
(category 1) and solar influx and shortwave absorption (category 2).

The radiation code is then called additionally twice for every "perturbation"-altitude $z_{\mathrm{p}}$ with a perturbation in the temperature and with a perturbation in the greenhouse gas concentration (in ppbv). This provides a change in the heating rate profile per change in temperature and greenhouse gas concentration, respectively at all perturbation altitudes: $H_{\mathrm{T}}\left(z, z_{\mathrm{p}}\right)$ in $\mathrm{s}^{-1}$ and $H_{\mathrm{G}}\left(z, z_{\mathrm{p}}\right)$ in $\mathrm{Ks}^{-1} \mathrm{ppbv}^{-1}$.

The heating profiles $H^{j}(z)$ for the individual categories $j$ depend on both the contributions of the two categories to the temperature $T^{j}(z)$ and the greenhouse gas concentration $G^{j}(z)$ :

$H^{j}(z)=H(z) \frac{\sum_{z_{\mathrm{p}}}\left(H_{\mathrm{T}}\left(z, z_{\mathrm{p}}\right) T^{j}\left(z_{\mathrm{p}}\right)+H_{\mathrm{G}}\left(z, z_{\mathrm{p}}\right) G^{j}\left(z_{\mathrm{p}}\right)\right)}{\sum_{z_{\mathrm{p}}}\left(H_{\mathrm{T}}\left(z, z_{\mathrm{p}}\right) T\left(z_{\mathrm{p}}\right)+H_{\mathrm{G}}\left(z, z_{\mathrm{p}}\right) G\left(z_{\mathrm{p}}\right)\right)}$.

Note that the magnitude in the perturbation has to be considered carefully in order to avoid impacts from numerical noise, if the perturbation was chosen too small and to avoid a too large deviation from the derivative if the perturbation was chosen too large.

\section{Singularities in the tagging formula}

The tagging method as described in Eq. (10) has an obvious disadvantage, since it may not be applicable in situation where $\boldsymbol{x}^{\mathrm{T}} \nabla F_{i}(\boldsymbol{x})$ becomes zero. Fortunately, this situation does not occur for many applications, e.g. for atmospheric chemistry or simple temperature tagging, as shown in the examples in Sects. 3.1-3.3. However, some singularities remain in these examples, namely if the state variables and concentration of species become zero. In these cases it can be argued that since no reaction occurs, the contributions of the tagged species to the individual species remain unchanged.

In general, $\boldsymbol{x}^{\mathrm{T}} \nabla F_{i}(\boldsymbol{x})$ means that the sensitivities of the term $F_{i}$ are fully balanced. Figure 1 gives an example for a two-dimensional situation, with two state variables $x_{1}$ and $x_{2}$. Here, the forcing term (thin isolines) is $F_{1}(\boldsymbol{x})=0.25-(\boldsymbol{x}-$ $\boldsymbol{a})^{2}$, with $\boldsymbol{a}^{\mathrm{T}}=(1 / 2,1 / 2)$. Hence the derivative is $\nabla F_{1}(x)=$ $-2(\boldsymbol{x}-\boldsymbol{a})$. For every $\boldsymbol{x}$ with $\boldsymbol{x}^{\mathrm{T}} \nabla F_{1}(\boldsymbol{x})=0$ (thick line) the sensitivity of the forcing term $F_{1}(\boldsymbol{x})$ with respect to $x_{1}$, which is $x_{1} \frac{\partial}{\partial x_{1}} F_{1}(x)$, is balanced by the sensitivity with respect to the second variable $x_{2}$ and the sum equals zero. Or in other words $\boldsymbol{x}$ is perpendicular to $\nabla F_{1}(\boldsymbol{x})$ (arrows).

If we replace the forcing term by $F_{1}(\boldsymbol{x})=x_{1} / x_{2}$ in this example, we obtain an extreme singularity. For this forcing term the denominator in the tagging equation becomes zero:

$$
\begin{aligned}
\boldsymbol{x}^{\mathrm{T}} \nabla F_{1}(\boldsymbol{x}) & =\left(x_{1}, x_{2}\right)\left(\frac{1}{x_{2}}, \frac{-x_{1}}{x_{2}^{2}}\right)^{\mathrm{T}} \\
& =\frac{x_{1}}{x_{2}}-\frac{x_{1}}{x_{2}}=0 .
\end{aligned}
$$

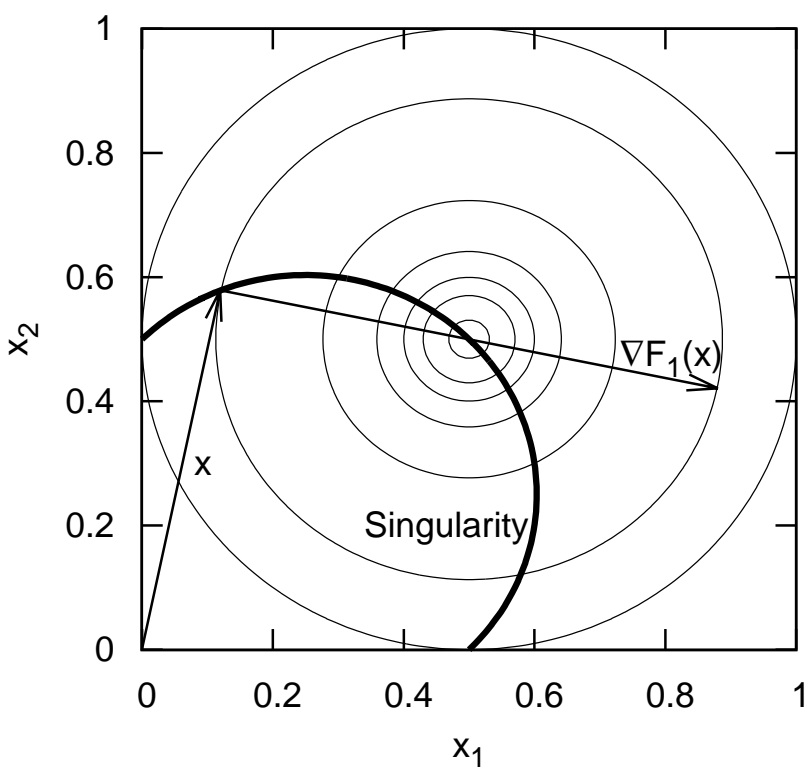

Fig. 1. Example of a singularity in the tagging method. The forcing term $F_{1}(\boldsymbol{x})$ is shown in thin lines with isolines $0 ., 0.1,0.2,0.23$, $0.24,0.245$, and 0.249 with a maximum in the middle. Thick lines indicate the location of the singularities $x \nabla F_{1}(x)=0$. The arrows give an example.

Hence, for individual cases, such as atmospheric chemistry, singularities can consistently be removed, by setting the respective forcing term to zero, with the argument that if no reaction occurs also no changes in the tagged species can occur. However, as the last extreme case shows, theoretically, there are constellations, where an application of the tagging formula is not possible.

\section{Comparison of diagnostical methods}

As shown in the previous section, tagging is a powerful methodology to provide more insights in functional chains of non-linear systems. Evidently, many previously used diagnostics also provided insights, however, with different aspects. Based on a simple non-linear system, a brief intercomparison is given here.

As a test-bed the following non-linear system is analysed:

$\dot{x}=P-x^{\alpha}=: G(x)$,

where $P>0$ is a production term, $x>0$ a state variable, and a non-linearity parameter $\alpha>0$. Note that this system is linear for $\alpha=1$. The results are summarized in Tab. 2 .

The production term has two sources $P_{1}>0$ and $P_{2}>0$ with $P_{1}+P_{2}=P$. The tagged variables $x_{1}$ and $x_{2}$, which resemble the impact of the individual sources on $x$ are 
$\dot{x}_{1}=P_{1}-x^{\alpha} \frac{x_{1}}{x}$

$\dot{x}_{2}=P_{2}-x^{\alpha} \frac{x_{2}}{x}$

The steady-state solutions are

$x^{\mathrm{s}}=P^{\frac{1}{\alpha}}$

${ }^{t} x_{1}^{\mathrm{s}}=P_{1} P^{\frac{1}{\alpha}-1}$

${ }^{t} x_{2}^{\mathrm{s}}=P_{2} P^{\frac{1}{\alpha}-1}$.

That means that the contribution of the production term $P_{1}$ to the state variable $x$ in steady-state is ${ }^{t} x_{1}^{\mathrm{s}}$.

Using the perturbation approach, the contribution $\mathrm{p}^{\mathrm{s}}$ and $\mathrm{p}_{x_{\mathrm{s}}}^{\mathrm{s}}$ of the source terms $P_{1}$ and $P_{2}$ to the steady-state solution $x^{\mathrm{s}}$ are calculated as

$$
\begin{aligned}
\mathrm{p}_{x_{1}} & =\frac{\partial x^{\mathrm{s}}}{\partial P} P_{1} \\
& =\frac{1}{\alpha} P^{\frac{1}{\alpha}-1} P_{1} \\
\mathrm{p}_{x_{2}} & =\frac{\partial x^{\mathrm{s}}}{\partial P} P_{2} \\
& =\frac{1}{\alpha} P^{\frac{1}{\alpha}-1} P_{2} .
\end{aligned}
$$

Obviously, the solutions are equal, i.e. ${ }^{t} x_{i}^{\mathrm{s}}={ }^{\mathrm{p}} x_{i}^{\mathrm{s}}(i=1,2)$ if and only if $\alpha=1$. In general, the factor between the two approaches is $\alpha$.

The decay-time or lifetime of the state variable $x$ (and also $\left.x_{i}\right)$ in steady-state is

$$
\begin{aligned}
\tau_{x} & =\frac{x^{\mathrm{s}}}{x^{\mathrm{s} \alpha}} \\
& =P^{\frac{1-\alpha}{\alpha}},
\end{aligned}
$$

whereas any perturbation to this steady state is approximately given by the linearized perturbation equation:

$$
\begin{aligned}
\dot{y} & =\frac{\partial}{\partial x} G\left(x^{\mathrm{s}}\right) y \\
& =-\alpha P^{\frac{\alpha-1}{\alpha}} y .
\end{aligned}
$$

The Jacobian is in this case a one dimensional derivative, only. The eigenvalue analysis is simple and provides one mode, namely, the perturbation life time $\tau_{y}$, which equals $\frac{1}{\alpha} P^{\frac{1-\alpha}{\alpha}}=\frac{\tau_{x}}{\alpha}$ and differs from the lifetime of $x$ by the factor $\alpha$. Or in other words, both life times are only equal in a linear system $(\alpha=1)$.

The contributions of $P_{i}$ to $x$ based on the tagging and perturbation approach can then be written as

$$
\begin{aligned}
{ }^{t} x_{i}^{\mathrm{s}} & =P_{i} \tau_{x} \\
{ }^{\mathrm{p}} x_{i}^{\mathrm{s}} & =P_{i} \tau_{y} .
\end{aligned}
$$

In the same way, the linearized perturbation equation can be derived:

$\frac{\partial}{\partial t}\left(\begin{array}{l}y_{1} \\ y_{2}\end{array}\right)=\left(\begin{array}{cc}\frac{\alpha P_{2}+P_{1}}{P} \tau_{x}^{-1} & \tau_{x}^{-1} \\ \tau_{x}^{-1} & \frac{\alpha P_{1}+P_{2}}{P} \tau_{x}^{-1}\end{array}\right)\left(\begin{array}{l}y_{1} \\ y_{2}\end{array}\right)$.

This analysis is hence a combination of an eigenvalue analysis, see e.g. in Prather (1998), with a tagging approach which allows for the analysis of how changes in $P_{1}$ and $P_{2}$ lead to changes in the chemical modes of the tagged species $x_{1}$ and $x_{2}$, respectively.

\section{Conclusions}

In this study, a tagging approach is presented, which allows the calculation of the contribution from individual processes or quantities to state variables for application in climatechemistry models. For these contributions, also called tagged quantities or species, differential equations are presented, which result from a partitioning of the differential equations of the primary - untagged - state variables. This partitioning is based on the sensitivity of each individual term of the right hand side forcing term with respect to the state variables.

This methodology is fully consistent with previous approaches presented in Grewe et al. (2010, 2012), which were derived with a different ansatz, a combinatorial approach. Four examples show how to apply this formalism to climatechemistry applications. One example shows how the sensitivities can be derived numerically based on model internal subroutines which were, in this case the radiation code, which provides heating rates.

An example of a very simple non-linear one-dimensional system shows how different analysis methods can be combined to obtain better insights. It shows that the lifetime of a species and its perturbation lifetime differ in general and coincide if and only if the system becomes linear. In analogy, the change in the contribution of a perturbed source term to the regarded state variable (= tagged state variable) differs in general from the change in the state variable itself. Again it coincides if and only if the system becomes linear.

Despite the large possibilities, which this methodology offers, there are limitations to its applicability, since it may include singularities. For some applications, such as for chemical tagging, the singularities arise from reactions, in which species are involved, which totally vanish. Since this implies that the reaction does not occur anymore, the right hand side of the tagging equation can be set to zero. This means that the involved tagged species remain unchanged, as the untagged primary species.

In a further theoretical example no consistent removal of these singularities could be found and in an extreme case the tagging formula is not applicable at all, since it consists of singularities, only.

Therefore, the examples show that this generalized tagging method is nicely applicable to a number of variables, such as 
atmospheric concentrations or temperature. The limitations show that a careful consideration of the possible singularities is necessary, when applying the tagging formula.

Acknowledgements. This work was performed during a sabbatical (DLR-Forschungssemester) at the National Center of Atmospheric Research (NCAR) in Boulder, CO and I am grateful for being given this opportunity and would like to thank all persons who made this possible. Thanks to A. Schady for internal review and helpful comments. This work was also partly funded by the EU-project REACT4C (WP2) and the DLR-internal project VEU.

Edited by: H. Tost

\section{References}

Butler, T. M., Lawrence, M. G., Taraborrelli, D., and Lelieveld, J.: Multi-day ozone production potential of volatile organic compounds calculated with a tagging approach, Atmos. Environ., 45, 4082-4090, 2011.

Emmons, L. K., Hess, P. G., Lamarque, J.-F., and Pfister, G. G.: Tagged ozone mechanism for MOZART-4, CAM-chem and other chemical transport models, Geosci. Model Dev., 5, 15311542, doi:10.5194/gmd-5-1531-2012, 2012.

Grewe, V.: Technical Note: A diagnostic for ozone contributions of various $\mathrm{NO}_{\mathrm{x}}$ emissions in multi-decadal chemistryclimate model simulations, Atmos. Chem. Phys., 4, 729-736, doi:10.5194/acp-4-729-2004, 2004.

Grewe, V.: A new method to diagnose the contribution of anthropogenic activities to temperature: temperature tagging, Geosci. Model Dev. Discuss., 5, 3183-3215, doi:10.5194/gmdd-5-31832012, 2012.
Grewe, V., Tsati, E., and Hoor, P.: On the attribution of contributions of atmospheric trace gases to emissions in atmospheric model applications, Geosci. Model Dev., 3, 487-499, doi:10.5194/gmd3-487-2010, 2010.

Grewe, V., Dahlmann, K., Matthes, S., and Steinbrecht, W.: Attributing ozone to $\mathrm{NO}_{\mathrm{x}}$ emissions: implications for climate mitigation measures, Atmos. Environ., 59, 102-107, 2012.

Gromov, S., Jöckel, P., Sander, R., and Brenninkmeijer, C. A. M.: A kinetic chemistry tagging technique and its application to modelling the stable isotopic composition of atmospheric trace gases, Geosci. Model Dev., 3, 337-364, doi:10.5194/gmd-3-337-2010, 2010.

IPCC: Climate Change 2007: The Physical Science Basis, Contribution of Working Group I to the Fourth Assessment Report of the Intergovernmental Panel on Climate Change, edited by: Solomon, S., Qin, D., Manning, M., Chen, Z., Marquis, M., Averyt, K. B., Tignor, M., and Miller, H. L., Cambridge University Press, Cambridge, UK and New York, NY, USA, 996 pp., 2007.

Maas, U. and Pope, S. B.: Simplifying Chemical Kinetics: Intrinsic Low-Dimensional Manifolds in Composition Space, Combust. Flame, 88, 239-264, 1992.

Prather, M. J.: Time Scales in Atmospheric Chemistry: Coupled Perturbations to $\mathrm{N}_{2} \mathrm{O}, \mathrm{NO}_{\mathrm{y}}$, and $\mathrm{O}_{3}$, Science, 279, 1339-1441, 1998.

Uherek, E., Halenka, T., Borken-Kleefeld, J., Balkanski, Y., Berntsen, T., Borrego, C., Gauss, M., Hoor, P., Juda-Rezler, K., Lelieveld, J., Melas, D., Rypdal, K., and Schmid, S.: Transport impacts on atmosphere and climate: land transport, Atmos. Environ., 44, 4772-4816, 2010.

Wang, Z. S., Chien, C.-J., and Tonnesen, G. S. J.: Development of a tagged species source apportionment algorithm to characterize three-dimensional transport and transformation of precursors and secondary pollutants, J. Geophys. Res., 114, D21206, doi:10.1029/2008JD010846, 2009. 